\title{
Versicherung der Schweizer Ärzte
}

Rolf O. Heimgartner, Direktor
Am 20. Mai 2006 fand die 80. ordentliche Delegiertenversammlung der Versicherung der Schweizer Ärzte unter dem Präsidium von Dr. med. J. M. Fritzsche im Hotel Splendide Royal in Lugano statt.

Anwesend waren 21 Delegierte sowie die Herren Johann Kobel, alt-stv. Direktor, Ernst \& Young AG, Bern; Jakob Burkhard, stv. Direktor, Ernst \& Young AG, Bern; Peter W. Möschler, Versicherungsmathematiker, Bern; Richard Schaller, Versicherungsmathematiker/Aktuar, Weissensberg (D), und Fritz Jakober, Vermögensverwalter, Glarus.

Aufgrund der leichten Belebung der Weltwirtschaft entwickelten sich die Aktienmärkte sehr erfreulich. Obwohl alle wichtigen Notenbanken eine restriktivere Geldpolitik betreiben, sanken die langfristigen Zinsen mit Ausnahme der USA noch einmal markant. Dies führte im vergangenen Jahr zu einem deutlichen Anstieg der Dollarwährung. Ebenfalls leicht höher wurden der Euro, das britische Pfund und der Yen gehandelt.

Die Aussichten für 2006 versprechen Positives für die Finanzmärkte, was wir im heutigen kontinuierlichen Zinsanstieg sehen. Sofern die weltweiten Krisengebiete nicht für negative Überraschungen sorgen. Der Ölpreis bleibt ebenfalls ein bedeutender Unsicherheitsfaktor.

Die Bilanzsumme nahm gegenüber dem Vorjahr um 23 Millionen auf 1041078 Millionen Franken zu.

Die Prämieneingänge sowie die Kapitalerträge erhöhten sich gegenüber dem Vorjahr.

Die Kapitalanlagen teilen sich auf in:

\begin{tabular}{|c|c|}
\hline Festverzinsliche Wertpapiere & Fr. 498721 000.- \\
\hline Aktien und Anlagefonds & Fr. $\quad 62841000 .-$ \\
\hline Anrechte Anlagestiftungen & Fr. 107964 000.- \\
\hline Liegenschaften & Fr. $170608000 .-$ \\
\hline Hypotheken & Fr. 120252 000.- \\
\hline Policendarlehen & $959000 .-$ \\
\hline Investitionskredite & Fr. $\quad 430000 .-$ \\
\hline Festgelder & Fr. $14000000 .-$ \\
\hline
\end{tabular}

Die versicherungstechnischen Rückstellungen erhöhten sich gegenüber dem Vorjahr um Fr. 23 082 000.- auf Fr. $1026147000 .-$.

Die Delegiertenversammlung genehmigt einstimmig den Geschäftsbericht mit der Bilanz und der Betriebsrechnung für das Jahr 2005 und erteilt dem Verwaltungsratsausschuss, dem Verwaltungsrat und der Direktion für das abgelaufene Geschäftsjahr Decharge.

Die Delegiertenversammlung stimmt einstimmig dem Antrag des Verwaltungsrates zu und beschloss das nach Gutschrift und Äufnung der Rückstellungen verbleibende Jahresergebnis von Fr. 581487.- wie folgt zu verwenden:

Zuweisung an die allgemeinen Reserven Fr. 580000 .

Zuweisung an den Hilfsfonds \begin{tabular}{l} 
Fr. $1487 .-$ \\
\hline Fr. $581487 .-$ \\
\hline
\end{tabular}

Für die Zeit von der Delegiertenversammlung 2006 bis zur Delegiertenversammlung 2007 wurden die folgenden Revisionsstellen gewählt:

- Herr Peter W. Möschler, Bern, als Versicherungsmathematiker;

- Ernst \& Young AG, Bern, als buchhaltungstechnische Revisionsstelle.

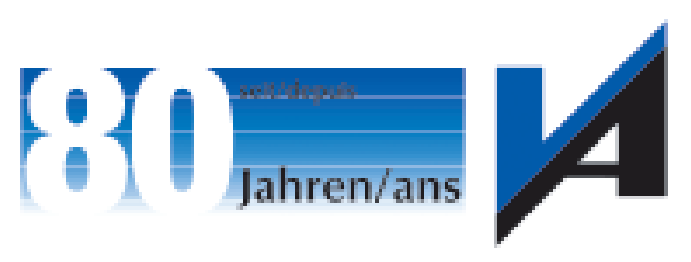

Korrespondenz:

Versicherung der Schweizer Ärzte

Länggassstrasse 8

CH-3000 Bern 9

Tel. 0313012555

Fax 0313025156

versa@versa.ch

www.versa.ch 